\title{
Nutrition Index is Maintained for Five Years after Pylorus-Preserving Pancreatoduodenectomy
}

\author{
Yuki Amano, Shinjiro Kobayashi, and Takehito Otsubo
}

(Received for Publication: August 20, 2020)

\begin{abstract}
[Background/Objective] We assessed trends in serum nutritional status markers and body mass index (BMI) to evaluate the long-term nutritional status in patients with pylorus-preserving pancreatoduodenectomy (PPPD). [Methods] From 240 patients who underwent PD from January 2005 to October 2014, we selected 45 patients who underwent PPPD and had survived without recurrence at 5-year postoperative follow-up. The following factors were analyzed preoperatively and at 2 weeks, and 1, 5 years, postoperatively: serum total protein (TP), serum albumin (Alb), and body mass index (BMI).

[Results] The mean TP value was $6.8 \mathrm{~g} / \mathrm{dL}$ preoperatively and 6.3 and $7.1 \mathrm{~g} / \mathrm{dL}$ at 2 weeks and 1 year after surgery, respectively $(\mathrm{P}<0.0001, \mathrm{P}=0.026$, respectively). TP remained greater than the preoperative values for up to 5 years. The mean Alb value was $4.1 \mathrm{~g} / \mathrm{dL}$ preoperatively, decreased to $3.5 \mathrm{~g} / \mathrm{dL} 2$ weeks after surgery, but returned to $4.15 \mathrm{~g} / \mathrm{dL} 1$ year after surgery and to $4.13 \mathrm{~g} / \mathrm{dL} 5$ years after surgery, values approximately equivalent to the preoperative value $\left(\mathrm{P}=0.134, \mathrm{P}=0.505\right.$, respectively). The mean preoperative BMI was $22.1 \mathrm{~kg} / \mathrm{m}^{2}$, decreased to $17.3 \mathrm{~kg} / \mathrm{m}^{2} 2$ weeks after surgery $(\mathrm{P}<0.001)$, and then rose to $19.0 \mathrm{~kg} / \mathrm{m}^{2}$ by 5 years after surgery $(\mathrm{P}=0.005)$, but remained within the standard range in 29 patients $(64.4 \%)$.
\end{abstract}

[Conclusion] Long-term nutritional status was adequately maintained postoperatively in patients who underwent PPPD.

\section{Key Words}

Blood proteins, body mass index, nutritional status, pancreatoduodenectomy, pylorus-preserving pancreatoduodenectomy

\section{Introduction}

Pancreatoduodenectomy (PD) is an invasive surgical technique. ${ }^{1,2)}$ During this procedure, the head of the pancreas and the duodenum, gall bladder and extrahepatic bile ducts are resected, leading to changes in the physiological function of gastric acid, pancreatic juice and bile, which affect postoperative nutritional status. ${ }^{3)}$ However, PD is indicated for intractable cancer of the pancreatic head and distal cholangiocarcinoma, and although these conditions are typically challenging to treat, their prognoses have improved due to advances in surgical dissection techniques and chemotherapy in recent years. ${ }^{4,5)}$ As long-term survival has now become feasible after PD, we must consider issues such as patient fragility, which are based on poor nutrition in patients who are already elderly and frail, in addition to the complication of sarcopenia. It remains unclear whether nutritional status can be maintained over the long term after PD. Some studies have evaluated postoperative nutritional status using serum albumin (Alb) level and body weight as indicators over a short period of about 6 months to 1 year after PD, but few reports have examined nutritional status after a long period of time. ${ }^{6-8)}$ In this study, we investigated the trends in serum nutritional status markers and body mass index (BMI) before and after surgery to evaluate the long-

Division of Gastrointestinal and General Surgery, St. Marianna University School of Medicine 
term nutritional status in patients who underwent pylorus-preserving pancreatoduodenectomy (PPPD).

\section{Patients and Methods}

Our patients were selected from 240 patients who underwent PD at St. Marianna University Hospital from January 2005 to October 2014. Of these patients, 193 underwent PPPD, and 45 survived without recurrence at 5 years after surgery. Ultimately, we selected these 45 patients for inclusion in the present study. We evaluated serum TP, serum Alb, and BMI, and the preoperative values and the values at 2 weeks, 1 year, and 5 years after the operation were compared by paired $t$-test for each item. In addition, the time course and standard error of the average values at these time points are shown in line graphs. Furthermore, for TP and Alb, subgroup analysis was performed in cases in which the preoperative values were either at or above or below the standard values.

Continuous variables are summarized by the mean \pm standard deviation, and discrete variables are summarized by number and percentage. The significance level of each test was 0.05 . Statistical analysis was performed using JMP Ver.14 (SAS Institute Japan Co., Ltd., Tokyo, Japan).

Five surgeons performed all surgeries, and the same surgical technique was used in all patients. Reconstruction after resection was accomplished using the modified Child method (Fig. 1) in which the elevated jejunum was anastomosed in the order of pancreas, bile duct and duodenum. The pancreatojejunostomy was undertaken by anastomosing two layers: the pancreatic duct was anastomosed to the jejunal mucosa using 5-0 absorbable suture, whereas the pancreatic parenchyma was sutured to the seromuscular layer of the jejunum using 4-0 non-absorbable suture. The cholangiojejunostomy was accomplished using interrupted sutures in all cases; 5-0 absorbable sutures were used if the bile duct diameter was $<15 \mathrm{~mm}$, and 4-0 absorbable sutures were used if the diameter was $\geq 15 \mathrm{~mm}$. The duodenojejunostomy was manually sutured using the Albert-Lembert anastomosis method. ${ }^{9)}$ All patients received a proton pump inhibitor (lansoprazole $30 \mathrm{mg}$ orodispersible tablets [Towa Pharmaceutical Co., Ltd., Osaka, Japan] taken once after breakfast) and continued treatment for 5 years after discharge. Patients were also administered digestive enzymes (stomilase granules, $3 \mathrm{~g}$ in three divided doses after breakfast, lunch and dinner [Sumitomo Pharmaceuticals Co., Ltd., Tokyo, Japan]) over the 5 years after they started oral intake. This

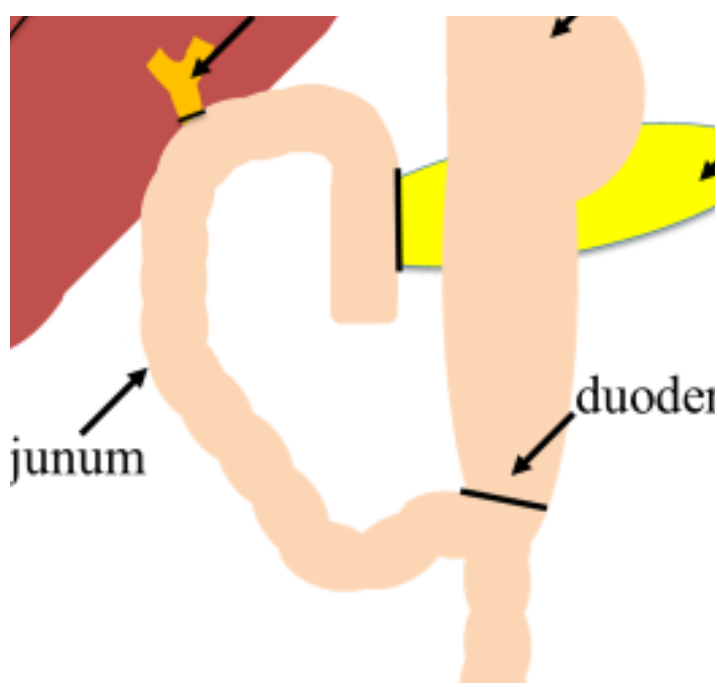

Fig. 1. In pancreatoduodenectomy, we always perform reconstruction by a modified Child method in which the elevated jejunum is anastomosed in the order of pancreas, bile duct, and gastrointestinal tract. (This figure is modified from a figure in Kobayashi et al. ${ }^{25)}$ )

study was approved by the St. Marianna University School of Medicine Internal Review Board (IRB No. 4827), and patient consent was obtained by allowing them to opt out of the study.

\section{Results}

The patients' characteristics are shown in Table 1 . There were 31 men and 14 women with a mean age of $68 \pm 9.2$ years at the time of surgery. Histopathology tests showed that 17 patients had pancreatic cystic tumors, 12 had cholangiocarcinoma, 6 had cancer of the pancreatic head, 6 had duodenal papilla carcinoma, 1 had a neuroendocrine tumor in the pancreatic head, and 3 had other tumors.

In the first year after surgery, the mean TP value was $7.1 \mathrm{~g} / \mathrm{dL}$, which was significantly higher than the preoperative value, and in the fifth year, the mean value was $7.0 \mathrm{~g} / \mathrm{dL}$, which was significantly higher than the preoperative value $(1$ year: $\mathrm{P}=0.005,5$ years: $\mathrm{P}=0.023)$. We further divided the patients into those with preoperative TP levels at or above the reference value ( $\geq 6.7 \mathrm{~g} / \mathrm{dL}, 25$ patients) and those below the reference value $(<6.7 \mathrm{~g} / \mathrm{dL}, 20$ patients) and performed further investigation. The mean preoperative TP value in patients with elevated preoperative levels $(\geq 6.7 \mathrm{~g} / \mathrm{dL}$ ) was $7.19 \mathrm{~g} / \mathrm{dL}$. This decreased significantly to a mean value of $6.53 \mathrm{~g} / \mathrm{dL} 2$ weeks after 
Table 1. Patient Characteristics

\begin{tabular}{|l|c|}
\hline \multicolumn{2}{|c|}{$\mathrm{n}=45$} \\
\hline Age (years) & $68 \pm 9.2$ \\
\hline Sex ratio (men:women) & $31: 14$ \\
\hline Disease & 12 \\
\hline Cholangiocarcinoma & 6 \\
\hline Cancer of the pancreatic head & 6 \\
\hline Duodenal papilla carcinoma & 17 \\
\hline Pancreatic cystic tumor & 1 \\
\hline Pancreatic neuroendocrine tumor & 2 \\
\hline Chronic pancreatitis & 1 \\
\hline Autoimmune thyroiditis & 26.7 \\
\hline Diabetes mellitus $(\%)$ & $160.7 \pm 9.2$ \\
\hline Preoperative body height $(\mathrm{cm})$ & $57.2 \pm 9.3$ \\
\hline Preoperative body weight $(\mathrm{kg})$ & $22.1 \pm 2.8$ \\
\hline Preoperative BMI $\left(\mathrm{kg} / \mathrm{m}^{2}\right)$ & $6.8 \pm 0.53$ \\
\hline Mean preoperative TP value $(\mathrm{g} / \mathrm{dL})$ & $4.1 \pm 0.32$ \\
\hline Mean preoperative Alb value $(\mathrm{g} / \mathrm{dL})$ &
\end{tabular}

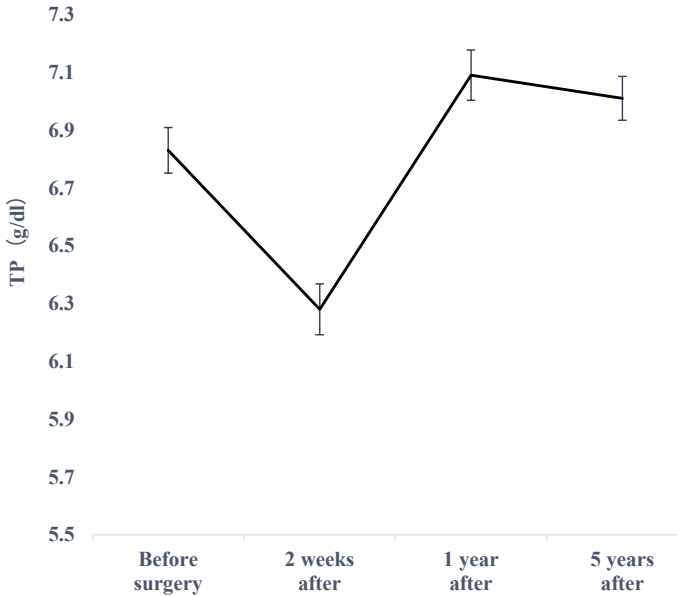

Fig. 2. Chronological trends in Total Protein (TP) in the 45 patients. The preoperative values were compared with the values at 2 weeks, 1 year and 5 years after surgery.

Paired $t$-test was used for comparison between two groups. Two weeks after surgery: $\mathrm{P}<0.0001$, 1 year later: $\mathrm{P}=0.005,5$ years later: $\mathrm{P}=0.023$. Error bars indicate standard errors.

surgery ( $\mathrm{P}<0.0001)$. The value improved to $7.1 \mathrm{~g} / \mathrm{dL}$ by 1 year after surgery, approximately equivalent to the preoperative value and remained stable up to 5 years. The mean preoperative TP value in the 20 patients with decreased preoperative levels $(<6.7 \mathrm{~g} / \mathrm{dL})$ was $6.34 \mathrm{~g} / \mathrm{dL}$. This decreased significantly to a mean value of $5.94 \mathrm{~g} / \mathrm{dL} 2$ weeks after surgery $(\mathrm{P}=0.009)$. The mean value increased to $7.02 \mathrm{~g} / \mathrm{dL}$ and exceeded the preoperative value at 1 year after surgery and was $6.84 \mathrm{~g} / \mathrm{dL}$ at 5 years after surgery, significantly increased compared to that before surgery $(\mathrm{P}<0.0001$ at 1 year, $\mathrm{P}=0.0003$ at 5 years; Fig. 3).

The mean preoperative Alb value was $4.1 \mathrm{~g} / \mathrm{dL}$ (Fig. 4). This value decreased significantly to a mean value of 3.52 weeks after surgery but increased to 4.2 and $4.1 \mathrm{~g} / \mathrm{dL}$ at 1 year and 3 years after surgery, respectively ( 1 year: $\mathrm{P}=0.1345,3$ years: $\mathrm{P}=0.504$ ). We further divided the patients into those with preoperative Alb levels at or above the reference value $(\geq 3.9 \mathrm{~g} / \mathrm{dL}, 33$ patients) and those below the reference value $(<3.9 \mathrm{~g} / \mathrm{dL}, 12$ patients $)$ and performed further investigation. The mean preoperative $\mathrm{Alb}$ value in patients with elevated preoperative levels was $4.2 \mathrm{~g} / \mathrm{dL}$. This value significantly decreased to $3.59 \mathrm{~g} / \mathrm{dL}(\mathrm{P}<0.0001)$. One year after surgery, it was $4.15 \mathrm{~g} / \mathrm{dL}$, and 5 years after the operation, it was 4.13 $\mathrm{g} / \mathrm{dL}$, which were not significantly different but maintained the same values as the preoperative value $(\mathrm{P}=$ $0.488,0.431$, respectively). The mean preoperative Alb value in patients with a decreased preoperative level was $3.69 \mathrm{~g} / \mathrm{dL}$. This decreased significantly to a mean value of $3.18 \mathrm{~g} / \mathrm{dL} 2$ weeks after surgery $(\mathrm{P}=$ $0.0018)$. It was $4.17 \mathrm{~g} / \mathrm{dL} 1$ year after surgery and $4.01 \mathrm{~g} / \mathrm{dL} 5$ years after surgery, which were significantly higher than the preoperative value $(1$ year: $\mathrm{P}=$ $0.0002,5$ years: $\mathrm{P}=0.0031)($ Fig. $\mathbf{5})$. 


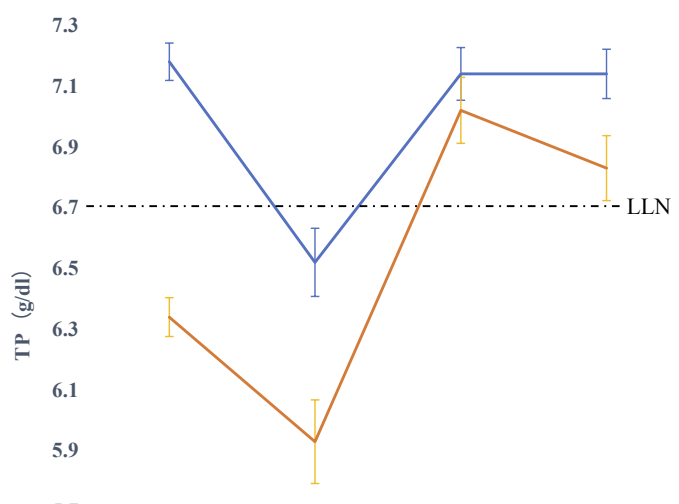

5.7

5.5

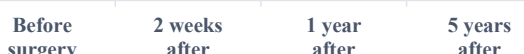

Chronological trends in serum TP with elevated values $(n=25)$

C Chronological trends in serum TP with decreased values $(n=20)$

Fig. 3. Chronological trends in total protein (TP) in the patients with TP at or above and below the standard value. Preoperative values were compared with the values at 2 weeks, 1 year and 5 years after surgery. Paired $t$-test was used for comparison between two groups. TP was above the standard value $(\geq 6.7 \mathrm{~g} / \mathrm{dL})$ in 25 patients. Two weeks after surgery: $\mathrm{P}<0.0001,1$ year later: $\mathrm{P}=0.636,5$ years later: $\mathrm{P}=0.613$. TP was below the standard value $(<6.7 \mathrm{~g} / \mathrm{dL})$ in 20 patients. Two weeks after surgery: $\mathrm{P}=0.0018,1$ year later: $\mathrm{P}=0.0002,5$ years later; $\mathrm{P}=0.0031$. Error bars indicate standard errors.

LLN: lower limit of normal

The mean preoperative BMI was $22.1 \mathrm{~kg} / \mathrm{m}^{2}$. It decreased to $17.3 \mathrm{~kg} / \mathrm{m}^{2} 2$ weeks after surgery $(\mathrm{P}=$ 0.0004), remained significantly decreased at 18.2 $\mathrm{kg} / \mathrm{m}^{2} 1$ year after surgery $(\mathrm{P}=0.0006)$, and then rose and remained at $19.0 \mathrm{~kg} / \mathrm{m}^{2} 5$ years after surgery. Although the BMI was lower than the preoperative value $(\mathrm{P}=0.005)$, in 29 patients $(\mathrm{P}=0.005) 64.4 \%)$ it had recovered to within the standard range (18.5$25 \mathrm{~kg} / \mathrm{m}^{2}$ ) according Japanese BMI data (Fig. 6).

\section{Discussion}

PD is a highly invasive surgical technique that can seriously effect postoperative gastrointestinal absorption. However, the present study showed that despite the fact that the Alb value was significantly lower than preoperative value at 2 weeks after surgery, the value improved and was approximately

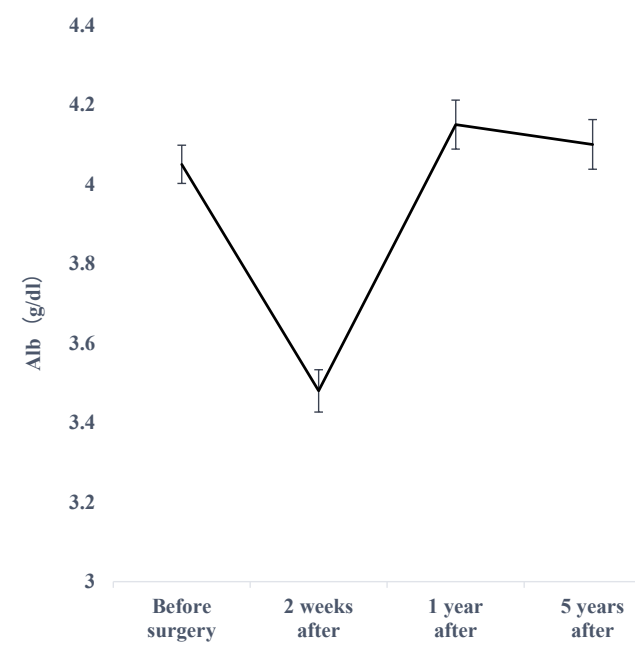

Fig. 4. Chronological trends in albumin (Alb) in the 25 patients with elevated values. Preoperative values were compared with the values at 2 weeks, 1 year and 5 years after surgery. Paired $t$-test was used for comparison between the two groups. Two weeks after surgery: $\mathrm{P}<0.0001,1$ year later: $\mathrm{P}=0.134,5$ years later: $\mathrm{P}=0.505$. Error bars indicate standard errors.

equivalent to the preoperative value by 1 year after surgery. Furthermore, the value did not decrease even after 5 years, suggesting that PPPD may maintain sufficient nutritional status even after a long period of time. BMI was significantly lower than the preoperative value even at 5 years after surgery. The BMI standard range for Japanese is $18.5-25 \mathrm{~kg} / \mathrm{m}^{2}$, and 5 years after surgery, it had recovered to $19.0 \mathrm{~kg} / \mathrm{m}^{2}$, which is within the standard range.

As several organs are removed during PD, it could be difficult to imagine that the exacerbation of postoperative nutritional status would improve over the long term. ${ }^{3}$ PPPD, which was first proposed in 1978 , is a surgical technique that preserves the stomach to retain function. ${ }^{10)}$ Compared to the classical technique (PD that includes pyloric resection), numerous reports have suggested that there is less weight loss and better nutritional status with PPPD ${ }^{11,12)}$ but these reports are for short-term periods of about 6 to 24 months after surgery, and PD and PPPD over the long-term postoperative course have not been compared. Meanwhile, reports have indicated that delayed gastric emptying frequently occurs as a complication in patients who have undergone PPPD,${ }^{13-15)}$ and surgical techniques that resect the py- 


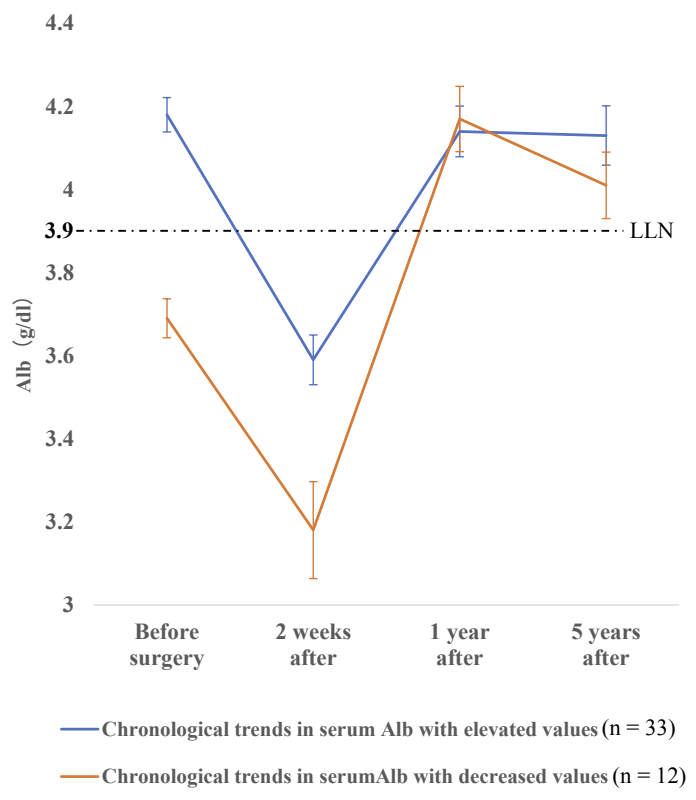

Fig. 5. Chronological trends in albumin (Alb) in the patients with Alb at or above and below the standard value. Preoperative values were compared with the values at 2 weeks, 1 year and 5 years after surgery. Paired $t$-test was used for comparison between two groups. Alb was above the standard value $(\geq 3.9 \mathrm{~g} / \mathrm{dL})$ in 33 patients. Two weeks after surgery: $\mathrm{P}<0.0001,1$ year later: $\mathrm{P}=0.488,5$ years later: $\mathrm{P}=0.431$. Alb was below the standard value $(<3.9 \mathrm{~g} / \mathrm{dL})$ in 12 patients. Two weeks after surgery: $\mathrm{P}=$ $0.0018,1$ year later: $\mathrm{P}=0.0002,5$ years later; $\mathrm{P}$ $=0.0031$. Error bars indicate standard errors.

LLN: lower limit of normal

loric sphincter are primarily performed to avoid this complication. ${ }^{16-18)}$ However, based on long-term postoperative follow-up, if the pyloric sphincter is inadvertently resected, there are concerns that the patient will be less able to store food in the stomach. During a distal gastrectomy, preservation of the pyloric sphincter significantly suppresses postoperative weight loss, and significantly fewer symptoms of dumping syndrome are also reported. ${ }^{19)}$ Even when performing $\mathrm{PD}$, we consider the original purpose of the stomach, the necessity to store food for long periods of time, which is why it is advisable to preserve the pyloric sphincter. At our hospital, we proactively preserve the pyloric sphincter in patients who have responded favorably to radical treatment. However, during this study, we only investigated PPPD in a sin-

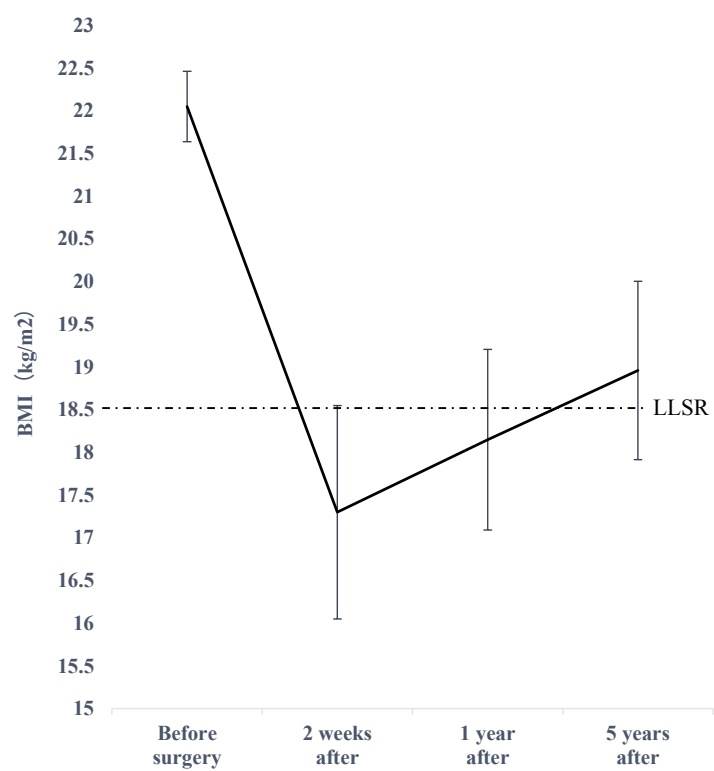

Fig. 6. Chronological trends in body mass index (BMI) in all 45 patients. Preoperative values were compared with the values at 2 weeks, 1 year and 5 years after surgery. Paired $t$-test was used for comparison between two groups. Two weeks after surgery: $\mathrm{P}=0.0004,1$ year later: $\mathrm{P}=0.006,5$ years later: $\mathrm{P}=0.05$. Error bars indicate standard errors.

LLSR: lower limit of standard range

gle arm, so we cannot make any conclusions about pyloric sphincter preservation. van Berge Henegouwen et al. compared patients who underwent standard PD with those who underwent PPPD at 15 months postoperatively and found that the PPPD patients had better outcomes, but they experienced weight loss.7) Melvin et al. examined body weight, BMI and serum Alb level in PPPD patients 5 years after surgery and reported a lack of improvement in nutritional status at this time. ${ }^{8)}$ We think that numerous multicenter comparisons using larger sample sizes will be necessary to determine the superiority of PPPD over standard PD in terms of long-term nutritional status.

There is an extreme decrease in both intra-pancreatic and extra-pancreatic endocrine function after standard PD, with the latter being responsible for the exacerbation of the nutritional status. ${ }^{20)}$ The BMI in the patients of the present study also decreased significantly. Accordingly, body weight or nutritional status may improve as a result of supplementation with pancreatic digestive enzymes in patients whose pancreatic fluid exhibits reduced enzymatic activ- 
ity. ${ }^{21,22)}$ Furthermore, the maintenance of nutritional status through supplementation with these digestive enzymes is reported to improve the prognosis for life expectancy, ${ }^{23)}$ and we believe that administration of digestive enzymes is essential after PD. It was possible to maintain the nutritional status of the present study patients as a result of this supplementation.

We expect the percentage of elderly patients who undergo PD to continue to increase as society continues to age. PD is a highly invasive procedure, so the risk to fragile elderly patients is high. ${ }^{24)}$ Furthermore, patients who undergo this procedure have a decreased capacity for gastrointestinal absorption, and as a result, fragile elderly patients may be unable to maintain their nutritional status and physical strength, although the present study suggests that the effects on long-term nutritional status are not as severe after PPPD. Particularly in terms of the TP and Alb levels, it was extremely interesting to see that the values at 5 years after surgery were equal to or higher than the preoperative values, but surgery was performed to improve nutritional status in patients with preoperative malnutrition. The present study divided patients into groups in which the previous value was at or above the standard value and groups in which the preoperative value was below the standard value. As a result, the 5-year mean value of the group below the standard value recovered to the same level as that of the group at or above the standard value. We believe that the nutritional state improved in the patients with malignant tumors who exhibited a tumor-bearing state or whose nutrition was impaired preoperatively due to the effects of conditions such as jaundice. The present study only used serum markers of nutrition to evaluate the nutritional state. To perform a more accurate evaluation of the nutritional state, it is necessary to evaluate both exocrine function and muscle mass, among other factors, and thus, we believe that further studies will be required going forward. In conclusion, this study showed that it is possible to maintain nutritional status during long-term follow-up after PPPD.

\section{Acknowledgments}

The authors are grateful to Prof. Tina Tajima of St. Marianna University School of Medicine for her insightful comments and meticulous language editing. The authors also thank Prof. Eisuke Inoue of Showa University for his help with statistical analysis.

\section{Conflicts of Interest}

The authors have nothing to disclose.

\section{References}

1) Schell MT, Barcia A, Spitzer AL, et al. Pancreaticoduodenectomy: volume is not associated with out come within an academic healthcare system. HPB Surg 2008; 2008: 1-6.

2) Kimura W, Miyata H, Gotoh M, et al. A pancreaticoduodenectomy risk model derived from 8575 cases from a national single-race population (Japanese) using a web-based data entry system: the 30-day and in-hospital mortality rates for pancreaticoduodenectomy. Ann Surg 2014; 259: 773-780.

3) Weimann A, Braga M, Harsanyi L, et al. ESPEN Guidelines on Enteral Nutrition: Surgery including organ transplantation. Clin Nutr 2006; 25: 224-244.

4) Uesaka K, Boku N, Fukutomi A, et al. Adjuvant chemotherapy of S-1 versus gemcitabine for resected pancreatic cancer: a phase 3, open-label, randomised, non-inferiority trial (JASPAC 01). Lancet 2016; 388: 248-257.

5) Glimelius B, Hoffman K, Sjödén PO, et al. Chemotherapy improves survival and quality of life in advanced pancreatic and biliary cancer. Ann Oncol 1996; 7: 593-600.

6) Kumagai Y, Fujioka S, Misawa T, et al. Comparison of short-term nutritional changes-between total pancreatectomy and pancreaticoduodenectomy. The Japanese Journal of SURGICAL METABOLISM and NUTRITION 2018; 52: 259-265.

7) van Berge Henegouwen MI, Moojen TM, van Gulik TM, et al. Postoperative weight gain after standard Whipple's procedure versus pyloruspreserving pancreatoduodenectomy: the influence of tumour status. Br J Surg 1998; 85: 922 926.

8) Melvin WS, Buekers KS, Muscarella P, et al. Outcome analysis of long-term survivors following pancreaticoduodenectomy. J Gastrointest Surg 1998; 2: 72-78.

9) Kobayashi S, Koizumi S, Hatori $T$, et al. The vertical array reconstruction technique in pylorus-preserving pancreatoduodenectomy. Dig Surg 2018; 35: 469-473.

10) Traverso LW, Longmire WP Jr. Preservation of the pylorus in pancreatico-duodenectomy. Surg 
Gynecol Obstet 1978; 146: 959-962.

11) Zerbi A, Balzano G, Patuzzo R, et al. Comparison between pylorus-preserving and Whipple pancreatoduodenectomy. Br J Surg 1995; 82: 975-979.

12) Niedergethmann M, Shang E, Soliman M, et al. Early and enduring nutritional and functional results of pylorus preservation vs classic Whipple procedure for pancreatic cancer. Langenbecks Arch Surg 2006; 391: 195-202.

13) van Berge Henegouwen MI, van Gulik TM, DeWit LT, et al. Delayed gastric emptying after standard pancreaticoduodenectomy versus pylorus-preserving pancreaticoduodenectomy: an analysis of 200 consecutive patients. J Am Coll Surg 1997; 185: 373-379.

14) Yeo CJ, Cameron JL, Sohn TA, et al. Six hundred fifty consecutive pancreaticoduodenectomies in the 1990s: pathology, complications, and outcomes. Ann Surg 1997; 226: 248-260.

15) Horstmann O, Markus PM, Ghadimi MB, et al. Pylorus preservation has no impact on delayed gastric emptying after pancreatic head resection. Pancreas 2004; 28: 69-74.

16) Nanashima A, Abo T, Sumida Y, et al. Comparison of results between pylorus-preserving pancreaticoduodenectomy and subtotal stomachpreserving pancreaticoduodenectomy: report at a single cancer institute. Hepatogastroenterology 2013; 60: 1182-1188.

17) Fujii T, Kanda M, Kodera Y, et al. Preservation of the pyloric ring has little value in surgery for pancreatic head cancer: a comparative study comparing three surgical procedures. Ann Surg Oncol 2012; 19: 176-183.

18) Kawai M, Tani M, Hirono S, et al. Pylorus ring resection reduces delayed gastric emptying in patients undergoing pancreatoduodenectomy: a prospective, randomized, controlled trial of py- lorus-resecting versus pylorus-preserving pancreatoduodenectomy. Ann Surg 2011; 253: 495501.

19) Nunobe S, Sasako M, Saka M, et al. Symptom evaluation of long-term postoperative outcomes after pylorus-preserving gastrectomy for early gastric cancer. Gastric Cancer 2007; 10: 167 172.

20) Pathanki AM, Attard JA, Bradley E, et al. Pancreatic exocrine insufficiency after pancreaticoduodenectomy: Current evidence and management. World J Gastrointest Pathophysiol 2020; 11: 20-31.

21) Kim H, Yoon YS, Han Y, et al. Effects of pancreatic enzyme replacement therapy on body weight and nutritional assessments after pancreatoduodenectomy in a randomized trial. Clin Gastroenterol Hepatol 2020; 18: 926-934.e4.

22) Nagai M, Sho M, Satoi S, et al. Effects of pancrelipase on nonalcoholic fatty liver disease after pancreaticoduodenectomy. J Hepatobiliary Pancreat Sci 2014; 21: 186-192.

23) Roberts KJ, Schrem H, Hodson J, et al. Pancreas exocrine replacement therapy is associated with increased survival following pancreatoduodenectomy for periampullary malignancy. HPB (Oxford) 2017; 19: 859-867.

24) Kobayashi S, Segami K, Hoshino H, et al. Risk factors for failure of early recovery from pancreatoduodenectomy despite the use of enhanced recovery after surgery protocols and a physical aging score to predict postoperative risks. J Hepatobiliary Pancreat Sci 2018; 25: 231-239.

25) Kobayashi S, Segami K, Hoshino H, et al. Twostage pancreatoduodenectomy in which pancreatojejunostomy performed in first stage for pancreatic trauma. JOP 2017; 18: 420-425. 